\title{
5 Risk measure choice
}

Tools designed to provide adequate risk measurements are needed by both decision making agents and regulatory agents, who require information about potential losses within a probabilistic framework. As such, the choice of a risk measure plays a central role in decision making in many areas including health, safety, environmental, adversarial and catastrophic risks [Cox Jr., L.A., 2013; MacKenzie, 2014]. Many different risk measures are available to practitioners, but the selection of the most suitable risk measure to be used in a given context is generally controversial. A key element in characterizing a risk measure is the underlying risk attitude that is assumed when this measure is used for risk assessment. Therefore, in selecting the best measure, the practitioner is concerned with how a particular measure matches up with the alternatives. However, this simple question only has a complex answer.

Consider the Value at Risk and the Tail Value at Risk, probably the most common risk measures used in assessing risk. Suppose $\alpha$ is the confidence level, which reflects the degree of tolerance to undesirable events. The $\operatorname{VaR}_{\alpha}(X)$ is the $\alpha$-quantile of loss $X$, while the $\operatorname{TVaR}_{\alpha}(X)$ averages quantiles ranging from the $\alpha$-quantile to the maximum (the $100 \%$-quantile) of $X$. Based on these definitions, it seems obvious that these two quantile-based risk measures can be directly compared in terms of their respective conceptions of risk using their associated confidence levels. For instance, the VaR provides for a concept of risk associated with a barrier, beyond which the decision maker assumes that catastrophe lies [Alexander and Sarabia, 2012]. A VaR at a $95 \%$ confidence level presents a lower resistance to undesirable events than a VaR at a $99 \%$ level. This also holds for $\operatorname{TVaR}_{\alpha}(X)$. Comparisons of VaR and TVaR measures can likewise be readily undertaken when their respective confidence levels are fixed and equal. Given an $\alpha$-confidence level, the $\operatorname{TVaR}_{\alpha}(X)$ is always greater or equal than the $\operatorname{VaR}_{\alpha}(X)$. However, a direct comparison cannot be made if the VaR and the TVaR risk measures 
have different confidence levels. For example, imagine a decision maker wishes to compare the implicit risk attitude of the $\operatorname{TVaR}_{95 \%}(X)$ and the $\operatorname{VaR}_{99 \%}(X)$. In this instance, it is not immediately obvious which of these two risk measures offers the greatest risk tolerance. Furthermore, if the decision maker wants to know the risk attitude of a measure other than that of these two quantile-based risk measures, comparisons are even less intuitive. Here, we focus on the family of distortion risk measures, where the VaR and TVaR can be understood as two particular cases. This chapter seeks to contribute to the study of attitudes towards risk in the assessment of risk. The study analyses the risk perception that is implicit when an agent applies a particular distortion risk measure. A battery of instruments is developed to facilitate the comparison of the risk attitude of distortion risk measures from both global and local perspectives. The results afford new elements for determining the suitability of a particular distortion risk measure in comparison with other available options. They also allow an agent to determine which risk measure provides the most risk tolerant behavior.

An illustrative example of the risk attitude characterization implicit in a distortion risk measure is included in this chapter. The European insurance regulatory framework serves as an excellent example of the choice of a compulsory risk measure, i.e. VaR99.5\% . However, insurers implement other choices in their internal tools. We show that, given a particular insurer's dataset, distortion risk measures other than that of the Value at Risk can provide the same risk estimates. However, if the insurer does choose a different risk measure, this chapter provides complementary tools for evaluating risk that can be used to understand its position in the European insurance or financial market, or even to benchmark it in relation to the mandatory risk assessment standard.

\subsection{Aggregate attitude towards risk}

The characterization of the implicit attitude towards risk in a given distortion risk measure is carried out by means of the analysis of the distortion function, which provides a precise portrait of the underlying risk position of a decision maker when selecting a particular risk measure for risk assessment.

Let us consider the mathematical expectation. As indicated previously in Section 3.2 of Chapter 3, the mathematical expectation can be understood as a distortion risk measure involving the identity function $i d$ as the associated distortion function. Figure 5.1 illustrates the distortion function associ- 
ated with the mathematical expectation. In other words, the mathematical expectation can be understood as the distortion risk measure used by agents when survival probabilities are not distorted (i.e. they are distorted by the identity function). So, the mathematical expectation can be associated with a risk neutral attitude of the agent.

Figure 5.1 Distortion function of the mathematical expectation

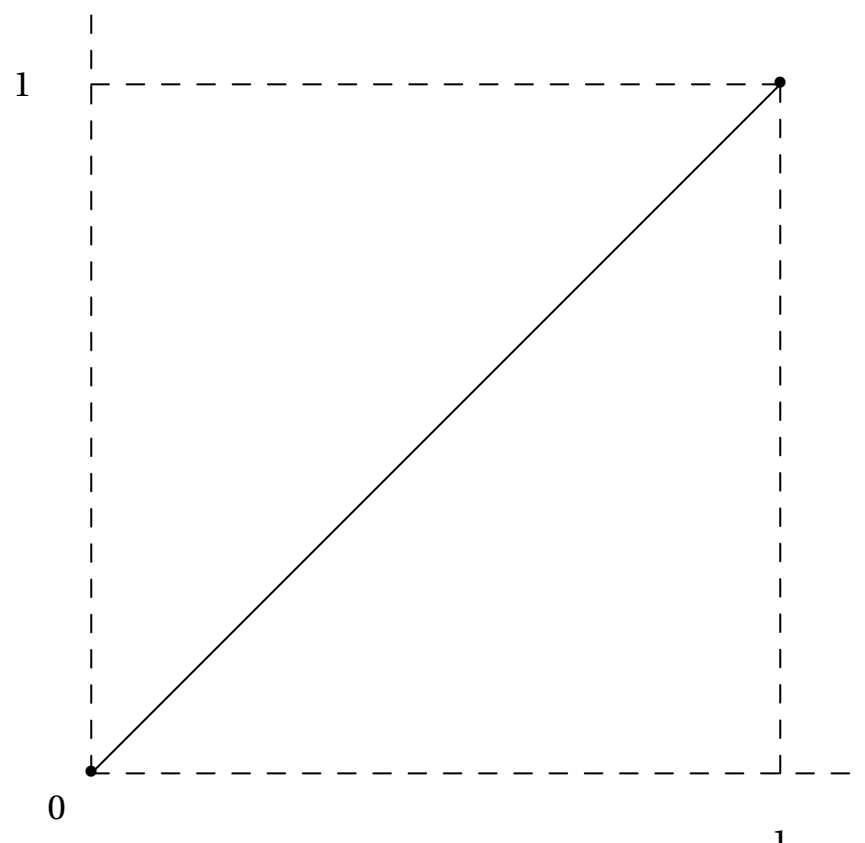

The area under the distortion function can be understood as an indicator of the aggregate risk attitude of an agent, with decision makers being classified as risk tolerant, risk neutral or risk intolerant. Note that we assume that a risk neutral agent would not distort the survival distribution function, so the associated distortion function linked to aggregated risk neutrality would be the $i d$ function. The area under the $i d$ function is one half and this value could be used as a benchmark of global risk attitude. A globally risk intolerant agent would make an upper distortion of the survival distribution in accumulated terms; thus, the area under $g$ for this agent would be larger than one half. Similarly, an agent would be globally risk tolerant if the area under $g$ was lower than one half.

The distortion functions of the Value at Risk and Tail Value at Risk with alpha confidence level are shown in Figure 5.2 and Figure 5.3, respectively. From 
Figure 5.2 it is straightforward to check that the area under the distortion function of the $\operatorname{VaR}_{\alpha}$ is $\alpha$. Similarly, from Figure 5.3 it is easy to observe that the area under the distortion function of the $\mathrm{TVaR}_{\alpha}$ is $\alpha+(1-\alpha) / 2$.

Figure 5.2 Distortion function of the $\operatorname{VaR}_{\alpha}$ risk measure

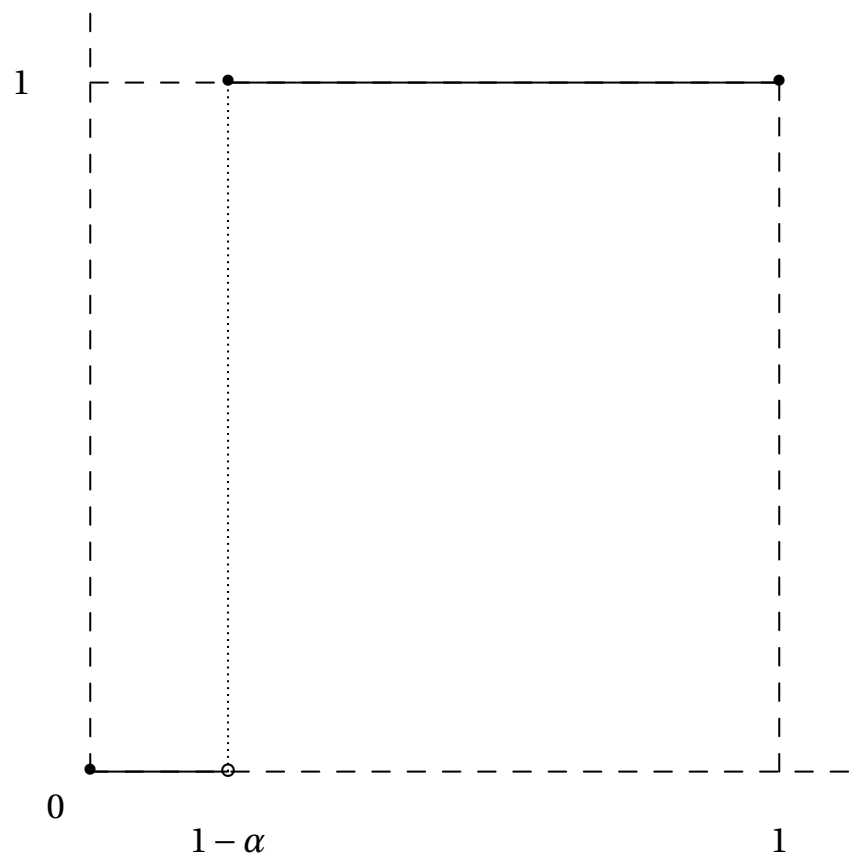

An agent would be globally risk neutral using either the $\operatorname{VaR}_{\alpha}$ measure with $\alpha=0.5$ or the $\operatorname{TVaR}_{\alpha}$ with $\alpha$ equal to zero. In terms of aggregate risk neutrality it would be equivalent to use either the mathematical expectation, or $\mathrm{VaR}_{50 \%}$ or $\mathrm{TVaR}_{0 \%}$. Note that $\mathrm{VaR}_{50 \%}$ is the median and $\mathrm{TVaR}_{0 \%}$ is the mathematical expectation. Similarly, an agent would be globally risk intolerant using either the $\operatorname{VaR}_{\alpha}$ with $\alpha>0.5$ or the $\mathrm{TVaR}_{\alpha}$ with a positive $\alpha$. On the contrary, an agent would be globally risk tolerant using the $\operatorname{VaR}_{\alpha}$ with $\alpha<0.5$. It is worthy to emphasize that under the definition of aggregate risk attitude followed in this chapter an aggregate risk tolerant agent would never use the TVaR risk measure, since the area under the distortion function is bounded in the interval $[0.5,1]$.

GlueVaR risk measures were introduced in Chapter 3 as a class of distortion risk measures. Recall that these measures are defined by means of four parameters $\alpha, \beta, \omega_{1}$ and $\omega_{2}$, such that $0<\alpha \leqslant \beta<1, \frac{\beta-1}{\beta-\alpha} \leqslant \omega_{1} \leqslant 1$ and $\omega_{1}+$ 
Figure 5.3 Distortion function of the $\mathrm{TVaR}_{\alpha}$ risk measure

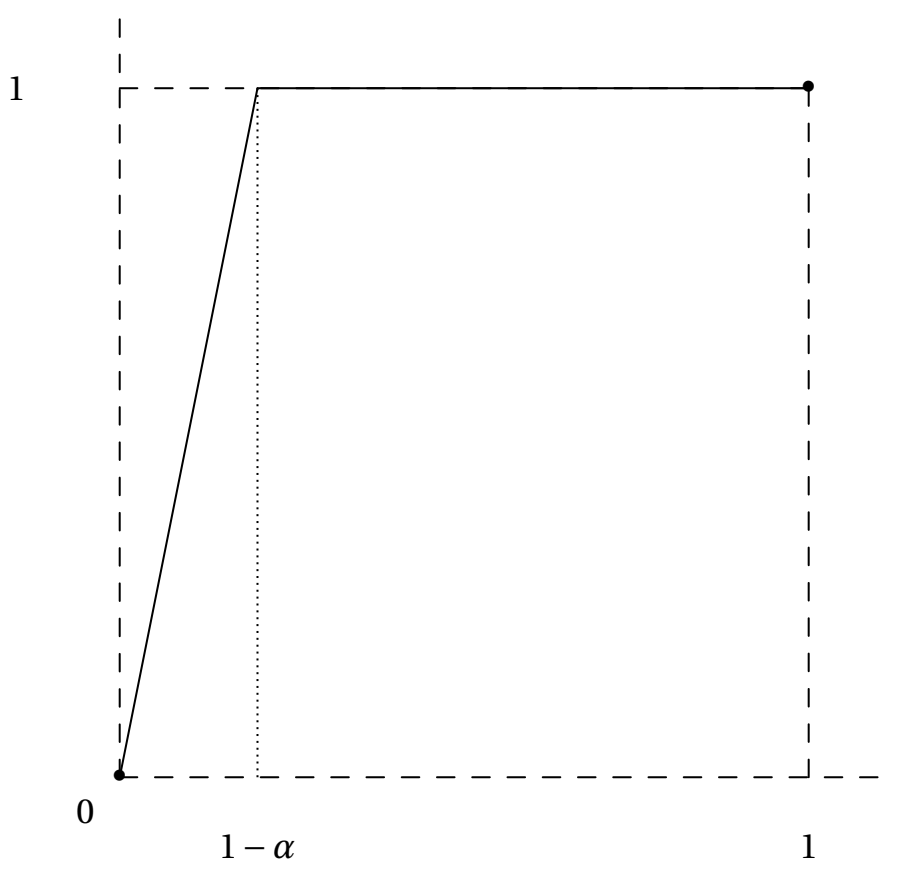

$\omega_{2} \leqslant 1$. This parametric flexibility of GlueVaR risk measures makes them useful in a variety of contexts, because each particular risk context could require a different set of parameter values. In the case of the GlueVaR $\mathrm{R}_{\beta, \alpha}^{\omega_{1}, \omega_{2}}$, the implicit global risk attitude would depend on the values of the four parameters that define the risk measure.

To conclude, in Belles-Sampera et al. [2013b] we introduced some relationships between distortion risk measures and aggregation operators. Aggregation functions (or operators) are mathematical functions used for combining information in many fields of human knowledge, as artificial intelligence, biology or economics [Torra and Narukawa, 2007]. Aggregation indicators are used to characterize the aggregation function [Belles-Sampera et al., 2014c, 2013c]. One of the most frequently used indicators is the degree of orness, which seeks to summarize the importance of each $i$ th-order statistic, $i=1, \ldots, n$, in the aggregation process associated with the Choquet integral with respect to a set function. This indicator provides some kind of level of preference inherent to such an aggregation function on a $[0,1]$ scale, where 0 represents the minimum and 1 the maximum order statistic (Dujmović [2006]; Fernández Salido and Murakami [2003]). In Belles-Sampera 
et al. [2016c] we showed that the degree of orness can be interpreted as an approximation of the area under the distortion function.

\subsubsection{Local risk attitude}

It is reasonable to suppose that decision makers do not worry about all random event losses in the same way. Decision makers frequently treat different random events distinctly (note that some of these events can represent benefits or affordable losses). While the area under the distortion function evaluates the accumulated distortion performed over the survival distribution function, it does not take into account which part of the survival distribution function was distorted. Clearly, from the perspective of a manager, distorting the survival probability in the right tail of the random variable linked to losses is not the same as distorting the probability in the left tail. Additionally, all distortion functions with an area equal to one half would be associated with global risk neutrality, where the $i d$ function is only a particular case.

In Figure 5.4 it is shown an example in which the size of the area under several distortion functions is the same. Obviously, these distortion functions have not associated the same risk attitude. In the case of the distortion function represented by a dotted line, survival probability values in the interval $[0,0.5]$ are overweighted and survival probability values in the interval $[0.5,1]$ are underweighted. So, relatively high losses are overrepresented (right tail) and relatively low losses are underrepresented. On the contrary, the distortion function represented by the solid line overweights relatively low losses and underweights high losses. Note that also the area under the diagonal, which is in fact the distortion function of the mathematical expectation, is the same.

Therefore, the global vision of risk embedded in a risk measure has to be completed with local information. One option open to us is to define the risk attitude in absolute terms. An absolute riskneutral agent is a decision maker that does not distort the survival probability and who, therefore, uses the $i d$ function as the associated distortion function, i.e. $g(u)=\mathrm{id}(u)=u$ for all $0 \leqslant u \leqslant 1$. An absolute risk intolerant agent is associated with a distortion function $g$ such that $g(u)>u$, for all $0 \leqslant u \leqslant 1$. And, similarly, an absolute risk tolerant agent has a distortion function $g$ such that $g(u)<u$, for all $0 \leqslant u \leqslant 1$. This definition of risk attitude is in absolute terms in the sense that the relationship of ordering between $g(u)$ and $u$ must be fulfilled in the whole range $[0,1]$. Note that these considerations lead to a more restrictive 
Figure 5.4 Example of distortion functions with the same area

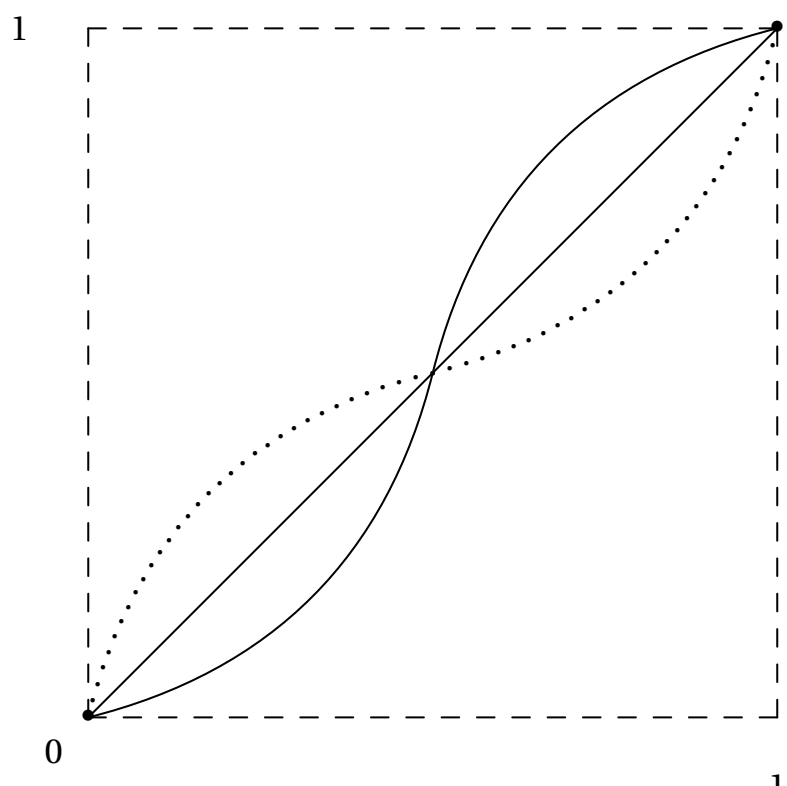

definition of risk attitude than that provided by the aggregate risk attitude. The definition of the absolute risk attitude implies that the implicit attitude of an agent is invariant over the range of values. Yet, there are no reasons as to why the agent should have a unique risk attitude across the whole domain. An agent's attitude to risk is likely to differ in accordance with the interval of loss under consideration. The risk attitude implicit in frequently used risk measures is not invariant; this is the case, for instance, of the $\mathrm{VaR}_{\alpha}$. When using the $\mathrm{VaR}_{\alpha}$, a risk intolerant attitude is associated with the interval $[1-\alpha, 1)$, but a risk tolerant attitude is associated with the interval $(0,1-\alpha)$. Thus, an homogeneous risk attitude cannot be linked to the $\operatorname{VaR}_{\alpha}$ risk measure throughout the domain.

Let us define a quotient function $Q_{g}$ from $(0,1]$ to $\mathbb{R}$, based on the distortion function $g$ associated with the risk measure, in order to characterize the local vision of risk. Let the function $Q_{g}$ be defined as the quotient between the distortion function $g$ and the identity function, $Q_{g}(u)=\frac{g(u)}{u}$ for all $0<u \leqslant 1$. The $Q_{g}$ allows the analysis of the agent's perception of risk at any point in the survival probability distribution. This quotient function provides a function of survival probabilities, $u$, which describes the distortion factor applied by $g$ at each $u$ level. The quotient $Q_{g}$ is a quantifier of 
the local risk tolerance of the agent at any point. The quotient value represents the relative risk attitude of the decision maker compared to that of an agent with a risk neutral attitude who is confident of the survival probability. An agent is risk neutral, risk tolerant or risk intolerant at point $u$ if $Q_{g}(u)$ is equal to, lower or higher than one, respectively.

A graphical analysis of the quotient function is proposed to investigate the risk attitude of the agent at any point in the survival distribution function when using a certain risk measure. An interesting characteristic is that the quotient function is bounded. Since the quotient function computes the ratio between the distorted survival probability and the survival probability, so $\frac{1}{u}$ is the maximum value attainable by this quotient function. In fact, the maximum risk intolerance frontier at the survival value equal to $u$ is achieved when $Q_{g}(u)=\frac{1}{u}$ (upper bound). Note that $Q_{g}$ takes non-negative values. The maximum local risk tolerance frontier is achieved when $Q_{g}(u)$ is equal to zero (lower bound). In addition, when the agent does not distort the survival probabilities, $Q_{g}(u)$ takes value equal to 1 (local risk neutrality line).

In Figure 5.5 bounds of the $Q_{g}$ are plotted. Upper and lower bounds are represented in the Figure 5.5 by a solid line. The local risk neutrality line is plotted by a dotted line. An agent's risk intolerance (tolerance) attitude emerges at point $u$ when the quotient function is bigger (smaller) than one. As the quotient function is bounded, we can deduce at any distorted survival value how far the value is from the maximum risk intolerance/tolerance.

The evaluation of the local risk appetite pattern of a manager using the $\mathrm{VaR}_{\alpha}$ and $\mathrm{TVaR}_{\alpha}$ is investigated. In Figure 5.6 the quotient functions associated with the $\mathrm{VaR}_{\alpha}$ and $\mathrm{TVaR}_{\alpha}$ are displayed, $Q_{\psi_{\alpha}}$ and $Q_{\gamma_{\alpha}}$ respectively.

If we focus our attention on the quotient function associated with the VaR, $Q_{\psi_{\alpha}}$, it can be seen that a radical risk attitude is implicit in the interval $[1-\alpha, 1)$, shifting to the opposite extreme in the interval $(0,1-\alpha)$. Indeed, a maximum risk intolerance is involved in $[1-\alpha, 1)$ and a maximum risk tolerance attitude is involved in $(0,1-\alpha)$. Some similarities are found when the quotient function associated with the TVaR is examined, $Q_{\gamma_{\alpha}}$. Two ranges involving a different risk attitude are also distinguished. Maximum risk intolerance is involved in the interval $[1-\alpha, 1)$ and a constant (non-boundary) risk intolerance attitude is involved in $(0,1-\alpha)$. In that interval, the quotient function value is farther to the maximum as closer is to zero the survival probability. Unlike the $\mathrm{VaR}_{\alpha}$, an absolute risk intolerance attitude is associated with the $\mathrm{TVaR}_{\alpha}$ because the quotient function is larger than one throughout the range $(0,1)$. 
Figure 5.5 Bounds of the quotient function

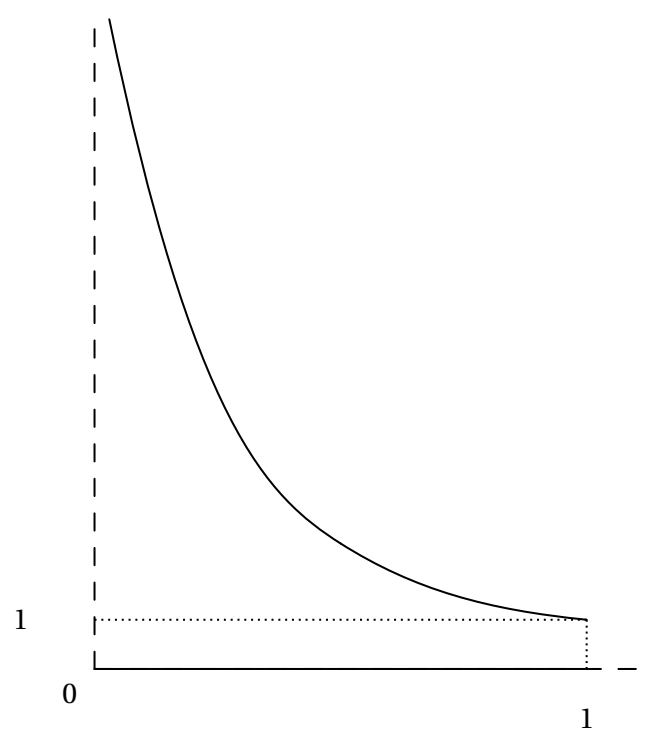

Figure 5.6 The quotient function of $\operatorname{VaR}_{\alpha}$ (left) and the quotient function of $\operatorname{TVaR}_{\alpha}$ (right).
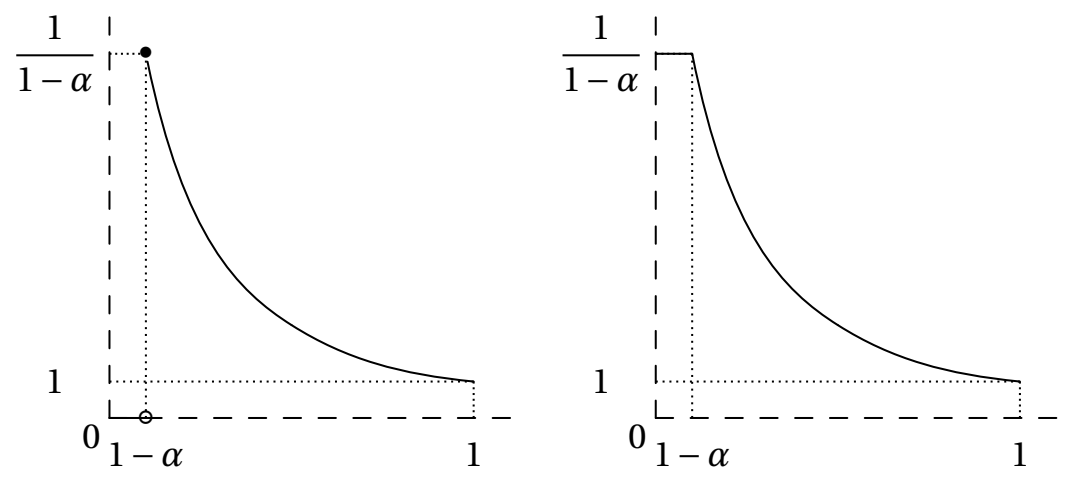

Note: Distortion functions only differ in terms of the interval $[0,1-\alpha)$. The quotient function of the mathematical expectation, $Q_{\mathrm{id}}$, is represented by a horizontal dotted line.

The quotient function of the GlueVaR $\mathrm{N}_{\beta, \alpha}^{\omega_{1}, \omega_{2}}$ is not plotted because the particular shape for the $Q_{\kappa_{\beta, \alpha} \omega_{1}, \omega_{2}}$ depends on the values of the four parameters that define the risk measure. However, as in the former two risk measures, 
a maximum risk intolerance is involved in the interval $[1-\alpha, 1)$ when the quotient function of the GlueVaR $\omega_{\beta, \alpha}^{\omega_{1}, \omega_{2}}$ is analyzed. However, more than just one attitude can be involved in the range $(0,1-\alpha)$. The high flexibility of GlueVaR $\omega_{\beta, \alpha}^{\omega_{1}, \omega_{2}}$ allows multiple attitudes towards risk to be implicit in the range $(0,1-\alpha)$, depending on the values of the remaining three parameters, $\beta, \omega_{1}$ and $\omega_{2}$.

In short, the quotient function $Q_{g}$ can be used to characterize the relative risk behavior of an agent at any point. The value of a quotient function at a particular point depends on the distortion function as well as on the original survival function. In other words, risk attitude in the quotient function is contemplated in the size of the distortion performed (the numerator) but also in the position in which this distortion is performed (the denominator). Note that the area under the quotient function provides similar information to that of the area under $g$, but expressed in terms of risk neutrality. Indeed, the area under the quotient function can be interpreted as the area under a weighted distortion function, where weights $(1 / u)$ are given to distorted values, i.e. $g(u) \cdot \frac{1}{u}$. Following this interpretation, greater weights are assigned to distortion function values associated with lower survival values. The areas under the quotient function of $\operatorname{VaR}_{\alpha}$ and $\mathrm{TVaR}_{\alpha}$ are $A\left(Q_{\psi_{\alpha}}\right)=-\ln (1-\alpha)$ and $A\left(Q_{\gamma_{\alpha}}\right)=1-\ln (1-\alpha)$, respectively. Similarly, the area under the quotient function of the GlueVaR $\mathrm{N}_{\beta, \alpha}^{\omega_{1}, \omega_{2}}$ is equal to

$$
A\left(Q_{\kappa_{\beta, \alpha}^{\omega_{1}, \omega_{2}}}\right)=\omega_{1}\left[1+\ln \left(\frac{1-\alpha}{1-\beta}\right)\right]+\omega_{2}-\ln (1-\alpha)
$$

Evaluating the area under the quotient function may be useful when analyzing the aggregate risk behavior in situations in which values of the distortion function are weighted, indicating that risk intolerance is negatively associated with the size of the survival values. Thus, the area under the quotient function can be interpreted as a weighted quantifier of the aggregate risk attitude, where an area equal to one indicates aggregate risk neutrality, an area larger than one indicates aggregate risk intolerance and an area lower than one indicates aggregate risk tolerance.

Example 5.1 (Obtaining risk attitudes of a RVaR risk measure). Taking into account the arguments presented in this chapter, the following corollaries of equivalence (4.13) proven in Section 4.3 of Chapter 4 may be stated: 
- The aggregate risk attitude of $\mathrm{RVaR}_{\alpha, \beta}$ can be obtained as the area under the distortion function plotted in Figure 4.1:

$$
\begin{aligned}
\frac{2-(a+b)-(1-a)}{2}+1-(2-(a+b)) & =\frac{1-b+2(a+b)-2}{2} \\
& =\frac{2 a+b-1}{2} \\
& =\frac{2-2 \alpha-\beta}{2}
\end{aligned}
$$

- The local risk attitude of $\mathrm{RVaR}_{\alpha, \beta}$ is given by the following quotient function:

$$
Q_{\mathrm{RVaR}}(u)= \begin{cases}0 & \text { if } u \in[0, \alpha] \\ \frac{u-\alpha}{u \beta} & \text { if } u \in(\alpha, \alpha+\beta] \\ \frac{1}{u} & \text { if } u \in(\alpha+\beta, 1]\end{cases}
$$

A numerical illustration of the results listed before is provided. Let us evaluate a $\mathrm{RVaR}_{0.1 \%, 5 \%}$ risk measure. It combines VaR at the $99.9 \%$ and TVaR at the $95 \%$. Parameters are $\alpha=0.1 \%$ and $\beta=5 \%$. From expression (5.1) the aggregate risk behavior of $\mathrm{RVaR}_{0.1 \%, 5 \%}$ is $\frac{2-0.2 \%-5 \%}{2}=\frac{194.8 \%}{2}=97.4 \%$. From expression (5.2), the specific risk attitude of $\mathrm{RVaR}_{0.1 \%, 5 \%}$ is described by the following quotient function:

$$
Q_{\mathrm{RVaR}}(u)= \begin{cases}0 & \text { if } u \in[0,0.1 \%] \\ \frac{1000 u-1}{50 u} & \text { if } u \in(0.1 \%, 5.1 \%] \\ \frac{1}{u} & \text { if } u \in(5.1 \%, 1]\end{cases}
$$

The equivalent GlueVaR risk measure to $\mathrm{RVaR}_{0.1 \%, 5 \%}$ is the one with parameters $a=99.9 \%, a+b-1=99.9 \%+95 \%-1=94.9 \%, h_{1}=0$ and $h_{2}=1$. In other words,

$$
\mathrm{RVaR}_{0.1 \%, 5 \%}=\text { GlueVaR}_{99.9 \%, 94.9 \%}^{0,1} .
$$

Given that a general expression for the overall or aggregate risk attitude behind a GlueVaR $\widehat{h}_{\widehat{\beta}, \widehat{\alpha}}^{\widehat{h}_{1}, \widehat{h}_{2}}$ risk measure is the following

$$
\widehat{\omega}_{1}\left(\frac{1+\widehat{\beta}-2 \widehat{\alpha}}{2}\right)+\widehat{\omega}_{2}\left(\frac{1-\widehat{\alpha}}{2}\right)+\widehat{\alpha}
$$


let us check if, using this formula for GlueVaR $\mathrm{R}_{99.9 \%, 94.9 \%}^{0,1}$ the $97.4 \%$ is recovered. From expressions (4.9):

$$
\begin{aligned}
\widehat{\alpha} & =a+b-1=94.9 \% \\
\widehat{\beta} & =a=99.9 \% \\
\widehat{\omega}_{1} & =\omega_{1}=-\frac{1-a}{1-b}=-\frac{0.1 \%}{5 \%}=-\frac{2}{100} \\
\widehat{\omega}_{2} & =\omega_{2}=\frac{2-(a+b)}{1-b}=\frac{194.9 \%}{5 \%}=\frac{194.9}{5} .
\end{aligned}
$$

Then

$$
\begin{gathered}
-\frac{2}{100}\left(\frac{1+99.9 \%-2 \cdot 94.9 \%}{2}\right)+\frac{5.1 \%}{5 \%}\left(\frac{1-94.9 \%}{2}\right)+94.9 \% \\
-\frac{2}{100}\left(\frac{10.1 \%}{2}\right)+\frac{5.1}{5}\left(\frac{5.1 \%}{2}\right)+94.9 \% \\
-0.101 \%+2.601 \%+94.9 \%=2.5 \%+94.9 \%=97.4 \% .
\end{gathered}
$$

\subsection{Application of risk assessment in a scenario involving catastrophic losses}

This section illustrates how the above findings can be applied in characterizing underlying risk attitudes. It is devised to highlight situations in which the implicit risk attitude linked to the VaR is unable to detect changes in potential catastrophic losses. We argue that the use of equivalent GlueVaR risk measures can be helpful in overcoming this drawback.

Suppose that the VaR with a confidence level $\alpha=99.5 \%$ is required to assess the regulatory capital under some regulatory framework. Note that the selection of the confidence level involves a trade-off between protection and competitiveness. The level of the protection could be reduced with low confidence levels. An increase in the confidence levels could involve higher economic reserves and, therefore, the protection would rise; however, this could also affect the competitiveness.

Risk managers may dislike using the VaR as a risk measure, because of the lack of risk-based information it provides on catastrophic losses. Indeed, two firms with marked differences in the sum of their potential losses in adverse scenarios may report the same risk value, even though they are not exposed to the same level of risk. As such, their disparities would go unobserved by decision makers. Moreover, the lack of subadditivity may well 
constitute another drawback. Alternatives to VaR99.5\% that take into account catastrophic losses can be considered by risk managers. Traditional approaches frequently lead to severely higher economic reserves. Managers need to find a risk measure that generates similar economic reserves than $V_{\text {Va.5\% }}$ for the overall risk faced by the insurance company and, additionally, they would like that the alternative risk measure provides risk-based information on catastrophic losses and that, hopefully, it satisfies appealing subadditivity properties.

\subsubsection{Calibration of GlueVaR parameters}

Our goal is to find the set of GlueVaR risk measures that return the same risk value that the $\mathrm{VaR}_{99.5 \%}$ in a particular context. So, we need to find the parameter values that define the GlueVaR ${ }_{\beta, \alpha}^{\omega_{1}, \omega_{2}}$ risk measures. All the steps required in calibrating GlueVaR risk measures are described here. The criterion followed in the calibration procedure is the need to obtain the same risk measure value with the GlueVaR risk measures as the one obtained with the $\mathrm{VaR}_{99.5 \%}$. Moreover, the selection of the risk measure is restricted to the subfamily of GlueVaR candidates that may satisfy that their distortion function is concave in $[0,1-\alpha)$. The strategy for calibrating the parameters is as follows:

- Minimum and maximum admissible values of the $\alpha$ and $\beta$ confidence levels have to be determined, $\alpha_{\min }$ and $\beta_{\max }$.

- Let us assume that $Z$ random variable represents the overall risk. A set of $d \times d$ constrained optimization problems is defined at this step:

$$
\begin{aligned}
& \mathrm{P}_{i, j}: \min _{\omega_{1}, \omega_{2}}\left|\mathrm{GlueVaR}_{\beta_{j}, \alpha_{i}}^{\omega_{1}, \omega_{2}}(Z)-\operatorname{VaR}_{99.5 \%}(Z)\right|, \\
& \text { subject to }\left\{\begin{array}{l}
0 \leqslant \omega_{1} \leqslant 1, \\
0 \leqslant \omega_{2}, \\
\omega_{1}+\omega_{2} \leqslant 1
\end{array}\right.
\end{aligned}
$$

where $i, j=1, \ldots, d, \alpha_{i}=\alpha_{\min }+\frac{i-1}{d-1}\left(\beta_{\max }-\alpha_{\min }\right)$ and $\beta_{j}=\alpha_{i}+$ $\frac{j-1}{d-1}\left(\beta_{\max }-\alpha_{i}\right)$. Flexibility rises with the number of partitions $d$, as do computational costs. Constraints are fixed to guarantee that the distortion function of the GlueVaR is concave in $\left[0,1-\alpha_{i}\right)$.

- An optimization algorithm should be used to solve this set of problems. If $\mathrm{P}_{i^{*}, j^{*}}$ represents the problem for which the minimum value of the objective function is reached and $\left(\omega_{1}^{*}, \omega_{2}^{*}\right)$ is the associated solution, then 
a GlueVaR ${ }_{\beta_{j^{*}, \alpha_{i}^{*}}^{*}, \omega_{2}^{*}}^{\omega^{*}}$ is found with its distortion function concave in [0,1$\alpha_{i^{*}}$ ) and gives similar risk values to those obtained with $\mathrm{VaR}_{99.5 \%}$ when applied to the overall risk of the company. $\mathrm{P}_{i, j}$ problems may not have solutions. Were this to be the case, then the optimization criteria would have to be revised, including a lower $\alpha_{\min }$, a higher $\beta_{\max }$ and/or a larger $d$.

- More than one GlueVaR solution is frequently found. Alternative combinations of parameter values return the same objective function value, or a value that differs insignificantly. In this situation, solutions could be ranked in accordance with the underlying risk attitude involved. Here, we propose ranking the solutions based on the value of the area under the distortion function associated with each optimal risk measure. With this goal in mind, degrees of orness are computed for (multiple) optimal GlueVaR $\beta_{\beta_{j^{*}}, \alpha_{i^{*}}^{*}}^{\omega_{1}^{*}, \omega_{2}^{*}}$ solutions. Two particular GlueVaR risk measures among the set of solutions are of special interest:

Lower-limit solution. Selection of the GlueVaR risk measure with the associated minimum area under the distortion function;

Upper-limit solution. Selection of the GlueVaR risk measure with the associated maximum area under the distortion function.

In other words, boundaries of the area size under distortion functions are detected. Optimal GlueVaR risk measures linked to boundaries reflect the extreme risk attitudes of agents when the random variable $Z$ is analyzed.

\subsubsection{Data and Results}

We are going to use the dataset used in previous chapters. It contains $X_{1}$, $X_{2}$ and $X_{3}$. Total claim costs are the sum of the three random variables, $Z=X_{1}+X_{2}+X_{3}$. So the aggregate risk faced by the insurer is the sum of the three random variables $X_{i}, i=1,2,3$. We assume that the insurer uses the $\mathrm{VaR}_{99.5 \%}$ as its risk measure.

Before dealing with the calibration of the GlueVaR risk measures, we first compute the $\operatorname{VaR}_{99.5 \%}(Z)$ and its associated area under its distortion function. The risk measure value is equal to $\operatorname{VaR}_{99.5 \%}(Z)=51.05$ and the area under its distortion function is equal to 0.995 . Let us now focus on the strategy used to calibrate the GlueVaR parameters. The following steps are performed to obtain GlueVaR risk measures that are comparable to the $\operatorname{VaR}_{99.5 \%}(Z)$ :

a) the minimum and maximum values of confidence levels are fixed at $90 \%$ and $99.9 \%$, i.e. $\alpha_{\min }=90 \%$ and $\beta_{\max }=99.9 \%$; 
b) the number of partitions is stipulated in $d=25$, so we deal with 625 optimization problems;

c) the empirical distribution function of total claim costs is used for the risk quantification, and, finally;

d) the outcome choice of the GlueVaR solutions are obtained using constroptim function from rootSolve library in $\mathrm{R}$.

A more complex calibration problem involving a modified random variable including catastrophic losses can be found in Belles-Sampera et al. [2016c]. We obtained a set of optimal GlueVaR risk measures that give the same risk value as the VaR99.5\% in this specific context. Thus, 192 optimal solutions were found. Once a set of GlueVaR risk measures has been obtained as feasible solutions, the areas under the distortion functions linked to each GlueVaR were computed to characterize the respective underlying aggregated risk attitude. The boundary values and the associated GlueVaR risk measures were identified. We should emphasize that the maximum area was equal to the area of the $\operatorname{VaR}_{99.5 \%}$. In fact, the optimal GlueVaR $\omega_{\beta, \alpha}^{\omega_{1}, \omega_{2}}$ solution with the highest area size was the GlueVaR with parameters $\alpha=99.5 \%, \beta=$ 99.9\% and $\omega_{1}=\omega_{2}=0$, and it holds that GlueVaR ${ }_{99.9 \%, 99.5 \%}^{0,0}=\operatorname{VaR}_{99.5 \%}$ (see expression (3.4)). In other words, given a certain risk value, the $\mathrm{VaR}_{\alpha}$ is the GlueVaR risk measure that presents the highest area under the associated distortion function of all the GlueVaR risk measures that return this value. Recall that the distortion function associated with the $\operatorname{VaR}_{\alpha}$ assigns one to survival values higher than $(1-\alpha)$ and zero to the rest.

The minimum area under the distortion function and the associated GlueVaR risk measure for the original dataset are reported in Table 5.1. Information about the underlying aggregate risk attitude of the agent can be inferred from the minimum area. Table 5.1 shows that, for this dataset, there exists an optimal GlueVaR risk measure for which the area of the associated distortion function is approximately o.949. Thus, this GlueVaR risk measure gives the same value as that given by $\operatorname{VaR}_{99.5 \%}$ when applied to $Z$, but, in aggregate terms, it involves a more moderate distortion of the original survival distribution function and, consequently, less aggregate risk intolerance.

The area under the distortion function should be complemented with the examination of the quotient function which allows the relative risk attitude at any point of the survival distribution to be analyzed. The quotient functions of risk measures associated with boundary areas in both scenarios are examined. All the quotient functions analyzed are located in the upper risk- 
Table 5.1 Optimal GlueVaR risk measure

\begin{tabular}{cc}
\hline & Original dataset \\
\hline$\alpha$ & 0.900 \\
$\beta$ & 0.999 \\
$\omega_{1}$ & 0.483 \\
$\omega_{2}$ & $1.035 \cdot 10^{-6}$ \\
$\omega_{3}$ & 0.516 \\
\hline Area under the & \\
distortion function & 0.949 \\
\hline
\end{tabular}

Note: Parameter values of the associated GlueVaR risk measure equivalent to $\mathrm{VaR}_{99.5 \%}$ and minimum area under the distortion function.

tolerance frontier in the range $[0.10,1)$. For ease of comparison, the quotient functions are rescaled and their left-tails are shown only in the range $[0,0.10]$ in Figure 5.7.

Notable differences can be observed in the relative risk attitudes locally implicit in the left-tail of the quotient functions. Let us first examine the quotient function of the GlueVaR risk measure that presents the maximum degree of orness (left), which is the same quotient function associated with the VaR $\mathrm{V}_{9.5 \%}$. The agent is most risk intolerant at any point of the interval $[0.5 \%, 1)$ and maximum risk tolerant at $(0,0.5 \%)$. This means, the quotient function is located in the upper frontier at $[0.5 \%, 1)$ and in the lower frontier at $(0,0.5 \%)$. When the GlueVaR risk measure that presents the minimum area is analyzed (right), the patterns of the left-tails of the quotient functions are undoubtedly different. An interesting finding is that the $Q_{g}(u)$ is not located within the boundaries at any point of the interval $(0,0.10)$. This means, the risk intolerant attitude is not maximized in the range $[0.5 \%, 0.10)$ but, on the contrary, the agent is more risk intolerant to catastrophic losses at $(0,0.5 \%)$ than when using $\mathrm{VaR}_{99.5 \%}$. 
Figure 5.7 Quotient functions of optimal solutions
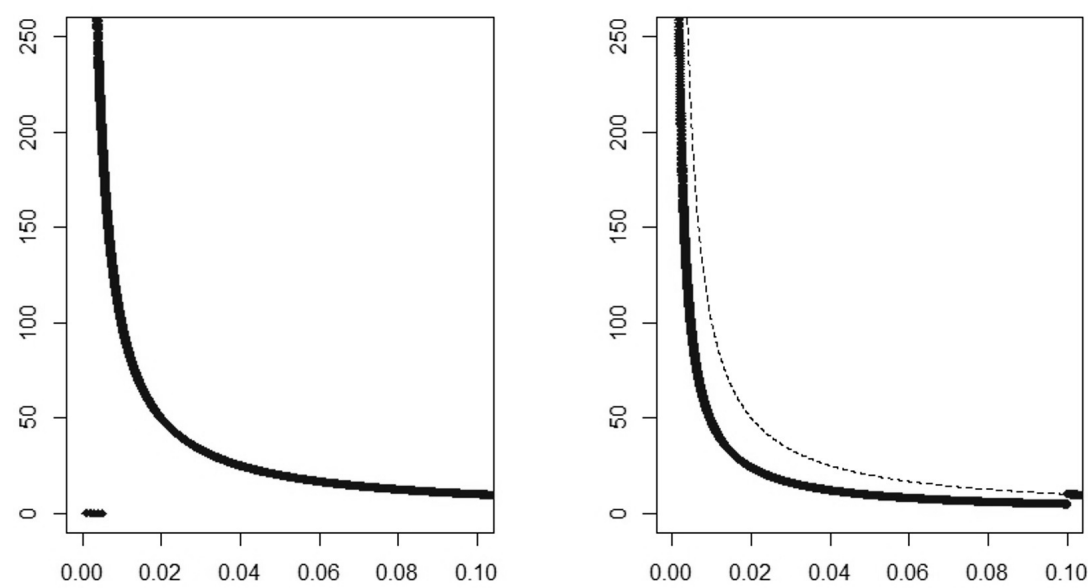

Note: Maximum (left) and minimum (right) areas under distortion functions of optimal GlueVaR risk measures. Dashed curve indicates the upper-bound quotient function curve, i.e. $\frac{1}{u}$ for all $0 \leqslant u \leqslant 1$.

\subsection{GlueVaR to reflect risk attitudes}

Distortion risk measures are widely used for assessing risk in a range of different contexts. As we have seen, the risk value obtained from such risk measures depends basically on the characteristics of the random variable, which are captured by the survival distribution function, and the associated distortion function. These two elements can be disaggregated and, here, our attention has focused on an analysis of the distortion function, which can be understood as a weighting function of survival probabilities. Thus, any risk attitude implicit in a distortion risk measure is to some extent attached to its distortion function.

The analysis of the risk attitude implicit in the Value at Risk illustrates that it is not sensitive to changes involving riskier scenarios. The reason for this is that the risk measure presents two extreme risk attitudes, i.e. maximum risk resistance in $[1-\alpha, 1)$ and maximum risk intolerance in $(0,1-\alpha)$. Here, we have shown that additional risk information may be obtained using comparable GlueVaR risk measures that are calibrated to report the same risk value as that of the $\operatorname{VaR}_{\alpha}$. The calibration procedure of GlueVaR parameters was not the primary focus of this chapter and it was not our intention to cover all the possible calibration criteria. However, two final remarks are 
worth recording. First, the procedure proposed depends necessarily on the risk measure of reference and also on the random variable. As such, the set of comparable GlueVaR risk measures differs when the random variable changes. This is not an unbridgeable drawback when requesting to the supervisory authorities authorization for changing the risk measure to a GlueVaR one, if decision makers are able to justify the GlueVaR selection process. For example, these measures may be used to analyze variations from one year to next in the implicit risk attitude of boundary cases when the $\mathrm{VaR}_{\alpha}$ is applied to assess the annual risk. Second, the $\operatorname{VaR}_{\alpha}$ was chosen as the risk measure of reference because of its application in practice, but the calibration strategy of the GlueVaR risk measures could easily be adapted to other risk measurement problems.

\subsection{Exercises}

1. Compare the area under the distortion function of the GlueVaR $85 \%, 50 \%$, GlueVaR $_{85 \%, 50 \%}^{0,1}$ and the GlueVaR ${ }_{85 \%, 50 \%}^{1 / 20,1 / 8}$. What do you can say in terms of aggregate risk attitude associated to these risk measures?

Hint: The heights $\left(h_{1}, h_{2}\right)$ equal to $(11 / 30,2 / 3),(0,1)$, and $(1 / 20,1 / 8)$ correspond to $\left(\omega_{1}=1 / 3, \omega_{2}=1 / 3\right),\left(\omega_{1}=-1 / 9, \omega_{2}=10 / 9\right)$ and $\left(\omega_{1}=\right.$ $\left.1 / 24, \omega_{2}=1 / 12\right)$, respectively.

2. Plot the quotient functions of previous GlueVaR risk measures. Discuss what additional information (if there are) in terms of local risk attitude is provided when the quotient function of these risk measures is analyzed.

3. Compute the area under the distortion functions of the $\mathrm{RVaR}_{1 \%, 5 \%}$ and $\mathrm{RVaR}_{5 \%, 1 \%}$.

- What do you can say in terms of aggregate risk attitude associated to these risk measures?

- Discuss their quotient functions in terms of the implicit local risk attitude.

4. Analyze the area under the distortion function and the quotient function of the Wang-Transform risk measure [Wang, 2002]. Discuss the relationship between $\alpha$ and the underlying risk attitude.

Hint: The distortion function of the Wang-Transform risk measure is defined as $g(u)=\Phi\left[\Phi^{-1}(u)-\lambda\right]$, where $u=S(x)$ and $\lambda=\Phi^{-1}(\alpha)$ with the security level $\alpha$. 